\title{
Tempo de coleta para determinação dos coeficientes de digestibilidade de nutrientes
}

\section{em equinos}

\author{
Collection time for determining the digestibility coefficients of nutrients in horses \\ Período de recolección para determinar los coeficientes de digestibilidad de los nutrientes en \\ caballos
}

Recebido: 18/12/2020 | Revisado: 23/01/2021 | Aceito: 26/01/2021 | Publicado: 02/02/2021

\author{
Clistenes Gomes de Oliveira \\ ORCID: https://orcid.org/0000-0002-1874-9613 \\ Universidade Federal de Sergipe, Brasil \\ E-mail: clismedvet80@hotmail.com \\ Gregório Murilo de Oliveira Júnior \\ ORCID: https://orcid.org/0000-0001-7391-272X \\ Universidade Federal de Sergipe, Brasil \\ E-mail: gregzootec@yahoo.com.br \\ Raquel Moura Silva \\ ORCID: https://orcid.org/0000-0002-9642-1779 \\ Universidade Federal de Lavras, Brasil \\ E-mail: raquelmourabr@yahoo.com.br \\ Paula Gomes Rodrigues \\ ORCID: https://orcid.org/0000-0003-1140-8899 \\ Universidade Federal de Sergipe, Brasil \\ E-mail: paulagrodrigues@hotmail.com \\ Claudson Oliveira Brito \\ ORCID: https://orcid.org/0000-0002-3339-8647 \\ Universidade Federal de Sergipe, Brasil \\ E-mail: claudson@ufs.br \\ Thailine Santana Santos \\ ORCID: https://orcid.org/0000-0001-7772-2130 \\ Universidade Federal de Sergipe, Brasil \\ E-mail: thai.zoo@hotmail.com \\ Douglas Melo de Souza \\ ORCID: https://orcid.org/0000-0002-2042-0745 \\ Universidade Federal de Sergipe, Brasil \\ E-mail: dmszootec@gmail.com \\ Camilla Mendonça Silva \\ ORCID: https://orcid.org/0000-0001-7391-272X \\ Universidade Federal de Sergipe, Brasil \\ E-mail: camillamsazoo@gmail.com
}

\begin{abstract}
Resumo
Objetivou-se avaliar três métodos de coleta de fezes utilizados para estimar os coeficientes de digestibilidade de nutrientes para equinos. Os tratamentos consistiram em três métodos de coleta de fezes, sendo eles: método de coleta total (controle); método da Liginina purificada LIPE $^{\circledR}$ com cinco dias de coleta de fezes (LIPE3) e o método da LIPE ${ }^{\circledR}$ com três dias de coleta de fezes (LIPE5). Para isto, foram utilizados seis equinos machos castrados, sem raça definida, com peso médio $442,3 \pm 33,3 \mathrm{~kg}$. Considerou-se três métodos de coleta e seis repetições. Foram calculados os coeficientes de digestibilidade da matéria seca, matéria orgânica, proteína bruta, extrato etéreo, hemicelulose, fibra em detergente neutro (CDFDN) e ácido (CDFDA) e carboidratos não estruturais (CDCNE). O CDFDN e CDFDA foram maiores $(\mathrm{P}<0,01)$ nos métodos LIPE $^{\circledR}$ (três e cinco dias de coleta). O CDCNE foi maior no método LIPE ${ }^{\circledR}$ com cinco dias de coleta. Além disso, a taxa de recuperação fecal do indicador LIPE ${ }^{\circledR}$ foram elevadas, independente do período de coleta. O método de coleta total de fezes e os métodos com coleta parcial de fezes durante três ou cinco dias com utilização de LIPE $^{\circledR}$ podem ser utilizados para se determinar os coeficientes de digestibilidade da matéria seca, matéria orgânica, extrato etéreo, proteína bruta e hemicelulose em equinos de forma confiável.
\end{abstract}

Palavras-chave: Análise de alimentos; Cavalos; LIPE; Coleta total; Nutrição.

\begin{abstract}
The objective of this study was to evaluate three fecal collection methods used to estimate nutrient digestibility coefficients in horses. Treatments consisted of three fecal collection methods, namely, total collection (control); use of purified, enriched lignin $\left(\right.$ LIPE $\left.^{\circledR}\right)$ as a marker with five days of fecal collection (LIPE 5); and use of LIPE ${ }^{\circledR}$ with three
\end{abstract}


days of fecal collection (LIPE 3). Six mixed-breed, castrated male horses with an average weight of $442.33 \pm 33.3 \mathrm{~kg}$, were used in the study. Three collection methods and six replications were considered. The digestibility coefficients of dry matter (DCDM), organic matter (DCOM), crude protin (DCCP), ether extract and neutral detergent fiber were calculated. However, the neutral detergent fiber digestibility coefficient was higher when the LIPE® methods (three and five collection days) were applied. The digestibility coefficient of non-structural carbohydrates was higher in the LIPE® method with five collection days. The total fecal collection method and the methods involving partial fecal collection during three or five days using LIPE $^{\circledR}$ can be safely adopted to determine the digestibility coefficients in horses.

Keywords: Food analysis; Horses; LIPE; Total collection; Nutrition.

\section{Resumen}

El objetivo fue evaluar tres métodos de recolección de heces utilizados para estimar los coeficientes de digestibilidad de nutrientes para caballos. Los tratamientos consistieron en tres métodos de recolección de heces, a saber: método de recolección total (control); Método de liginina purificada LIPE ${ }^{\circledR}$ con cinco días de recolección de heces (LIPE3) y método LIPE ${ }^{\circledR}$ con tres días de recolección de heces (LIPE5). Para ello se utilizaron seis machos castrados de raza mixta con un peso promedio de 442,3 $\pm 33,3 \mathrm{~kg}$. Se consideraron tres métodos de recolección y seis repeticiones. Se calcularon los coeficientes de digestibilidad de materia seca, materia orgánica, proteína cruda, extracto etéreo, hemicelulosa, fibra detergente neutra (CDFDN) y ácido (CDFDA) y carbohidratos no estructurales (CDCNE). El CDFDN y CDFDA fueron mayores ( $\mathrm{P}<0.01$ ) en los métodos LIPE® (tres y cinco días de recolección). La CDCNE fue mayor en el método LIPE® con cinco días de recolección. Además, la tasa de recuperación fecal del indicador LIPE ${ }^{\circledR}$ fue alta, independientemente del período de recolección. El método de recogida total de heces y los métodos con recogida parcial de heces durante tres o cinco días utilizando LIPE $^{\circledR}$ se pueden utilizar para determinar los coeficientes de digestibilidad de materia seca, materia orgánica, extracto etéreo, proteína bruta y hemicelulosa en caballos de forma fiable.

Palabras clave: Análisis de alimentos; Caballos; LIPE; Colección total; Nutrición.

\section{Introdução}

A digestibilidade dos nutrientes das dietas estima à porção do alimento consumido pelo animal passível de ser digerido e absorvido no trato gastrointestinal (Moss et al., 2017). O conhecimento desses coeficientes permite formular dietas mais precisas, com boa conversão dos nutrientes exógenos em ganhos produtivos.

Assim, o método de coleta total de fezes tem sido largamente utilizado para estimar os coeficientes de digestibilidade dos nutrientes de uma dieta, e consiste em rigoroso controle da quantidade de alimento ingerido e de fezes produzidas pelo animal, sendo muito bem aplicado para animais em confinamento (Silva et al., 2018). Associado a este método, pode-se utilizar indicadores indigestíveis na dieta, com o objetivo de se determinar com maior segurança o período inicial e final da coleta de fezes, prática essencial para obter resultados confiáveis (Moss et al., 2017). Os indicadores demonstram a quantidade de alimentos ou nutriente consumido, mensuraram a taxa de passagem dos alimentos, e permite estimar a digestibilidade total ou parcial de um determinado alimento (Valentini et al., 2012).

Nos últimos anos o indicador LIPE $^{\circledR}$ (lignina purificada e enriquecida) vem sendo utilizado para auxiliar na determinação dos coeficientes de digestibilidade de nutrientes em equinos (Moss et al., 2017; Figueiredo et al., 2019), e quando comparado com o marcador externo óxido crômico, demonstrou não haver diferenças entre os indicadores (Lazentta et al., 2009), possibilitando assim, seu uso na determinação de coeficientes de digestibilidade para equinos. Além disso, o uso da LIPE® permite reduzir o período de amostragem, obtendo resultados confiáveis, menor manipulação das fezes e otimização do tempo, pois a coleta é realizada uma vez ao dia (Soares et al., 2011). Neste método, a amostra não é destruída após a análise para sua dosagem na espectroscopia no infravermelho (Saliba, 2005), o que permite manter a integridade da amostra para análises posteriores, se necessário.

O uso da LIPE $^{\circledR}$ com cinco dias de coleta de fezes foi testada por meio das técnicas in vivo em comparação ao método de coleta total de fezes, com o objetivo estimar produção fecal, consumo e digestibilidade sem demonstrar diferenças significativas entre os métodos de coleta (Saliba, 2005; Vasconcellos et al., 2007; Lazentta et al., 2009; Moss et al., 2017; Garcia et al. 2014). Contudo, pesquisas com o uso do indicador durante um período menor de coleta, em especial com três dias, são inexistentes, fato que pode viabilizar redução dos custos sem afetar a eficiência e confiabilidade do método. 
Assim, a precisão dos indicadores em determinar a digestibilidade dos nutrientes da dieta pode influenciar na escolha das metodologias e, consequentemente, mudar as estimativas de produção fecal, consumo e digestibilidade (Soares et al., 2011). Por isso, torna-se essencial avaliar os indicadores e as metodologias de aplicação com a finalidade de determinar de maneira confiável os coeficientes de digestibilidade, em especial a LIPE ${ }^{\circledR}$.

Diante do exposto, o estudo tem como hipótese demonstrar que o indicador LIPE ${ }^{\circledR}$ com três dias de coleta pode ser aplicado de maneira confiável a fim de avaliar a digestibilidade de nutrientes em dietas para equinos. Sendo assim, objetivouse com avaliar três métodos de coleta de fezes utilizados para estimar os coeficientes de digestibilidade de nutrientes para equinos.

\section{Metodologia}

O projeto foi submetido e aprovado pelo Comitê de Ética em Experimentação Animal (CEPAP) da UFS, conforme protocolo no01/2017. O experimento foi realizado no Esquadrão da Polícia Montada do Estado de Sergipe (EPMON), na cidade de Aracaju/SE. Os animais foram alojados em baias individuais de alvenaria, com área útil disponível de $16 \mathrm{~m}^{2} / a n i m a l$, com piso de concreto revestido de borracha e declive de $10 \%$ para o escoamento de urina. As baias foram equipadas com bebedouro de alvenaria e três comedouros individuais, para o fornecimento do alimento concentrado, volumoso e para o sal mineral.

As temperaturas máximas e mínimas, bem como a umidade relativa foram coletadas diariamente utilizando-se termômetros de máxima e mínima e psicrômetro colocados no centro de uma baia na altura correspondente à dos animais. As temperaturas médias máxima e mínima aferidas durante o período do experimento foram $26,29 \pm 0,85^{\circ} \mathrm{C}$ e $24,59 \pm 0,92^{\circ} \mathrm{C}$, respectivamente. A umidade relativa do ar foi de 78,6\%.

Os tratamentos consistiram em três métodos de coleta de fezes: Método 1 (Controle) - Coleta total de fezes por três dias consecutivos; Método 2 - LIPE 3 - três dias consecutivos de coleta parcial das fezes e; Método 3 - LIPE 5 - cinco dias consecutivos de coleta parcial das fezes. Para isto, foram utilizados 6 equinos machos castrados, sem raça definida, com peso

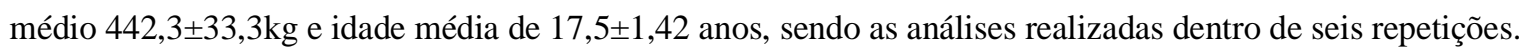

Para o método de coleta total de fezes (Método 1), aproximadamente 200g de fezes foram coletadas imediatamente após cada excreção durante três dias, diretamente do piso baia, sem que houvesse contaminação direta com solo, as quais representavam aproximadamente $15 \%$ do total das fezes coletadas. A cada 24 horas, todo volume fecal de cada animal foi pesado, inclusive as alíquotas coletadas para análise laboratoriais. As amostras obtidas ao longo de três dias, foram homogeneizadas e congeladas a $-10^{\circ} \mathrm{C}$.

Para o método da LIPE $^{\circledR}$, o indicador externo LIPE $^{\circledR}$ foi ofertado 48 h antes do início das coletas, em forma de cápsulas (500 mg/animal/dia) garantindo a ingestão total do indicador, junto com uma alíquota de $200 \mathrm{~g}$ do concentrado ofertado no período da tarde, (Figueiredo et al., 2019). No método LIPE 3, os animais consumiram o indicador uma vez ao dia durante o período de adaptação de $48 \mathrm{~h}$ e nos três dias de coleta parcial de fezes, totalizando cinco dias de administração do indicador, adaptando a técnica desenvolvida por Lanzetta et al. (2009). Contudo, no tratamento do indicador LIPE 5, os animais consumiram o indicador uma vez ao dia durante o período de adaptação de $48 \mathrm{~h}$ e nos cinco dias de coleta parcial de fezes, totalizando sete dias de administração do indicador.

Durante o ensaio de digestibilidade foi ofertado feno de Tifton 85-Cynodon spp. como alimento volumoso e ração comercial peletizada (ProequiMelaçada ${ }^{\circledR}$ ) como alimento concentrado, bem como água e sal mineral (Centauro $80^{\circledR}$ ) à vontade. Foi adotada a relação de 29:71 entre o alimento concentrado e volumoso, respectivamente, sendo considerado o consumo de

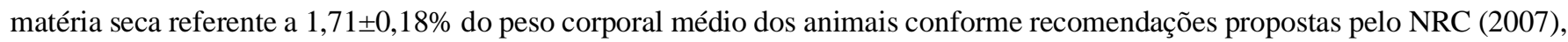
para cavalos adultos em manutenção.

A quantidade $(\mathrm{kg})$ de alimentos oferecidos diariamente aos animais, com base na matéria seca foi de 7,56 $\pm 0,87 \mathrm{~kg} / \mathrm{dia}$, deste $2,18 \pm 0,10 \mathrm{~kg}$ do concentrado comercial e $5,39 \pm 0,80 \mathrm{~kg} /$ dia de volumoso. Os animais receberam o alimento volumoso em 
três porções, sendo 1/3 às 7:00 horas, 1/3 às 11:00 horas e a última fração às 18:00 horas; o alimento concentrado foi fornecido em duas etapas, sendo metade às 04:00 horas e a outra metade às 14:30 horas. As amostras do alimento concentrado e volumoso foram coletadas para análise da composição química, de acordo com as recomendações de AOAC (2012) e encontram-se apresentados na Tabela 1 .

Tabela 1 - Composição química dos componentes da dieta oferecidos diariamente aos equinos, com base na matéria seca.

\begin{tabular}{lcc}
\hline \multirow{2}{*}{ Nutriente } & \multicolumn{2}{c}{ Alimentos } \\
\cline { 2 - 3 } & Concentrado $^{1}$ & Feno de Tifton $^{\circ}$ \\
Matéria seca (\%) & 90,79 & 86,63 \\
Matéria orgânica (\%) & 78,06 & 88,15 \\
Matéria Mineral (\%) & 21,94 & 11,85 \\
Extrato etéreo (\%) & 4,96 & 1,29 \\
Proteína bruta (\%) & 12,26 & 6,36 \\
FDN (\%) & 45,32 & 71,51 \\
FDA (\%) & 14,66 & 37,8 \\
Hemicelulose (\%) & 30,66 & 37,7 \\
CNE $^{2}(\%)$ & 15,52 & 8,99 \\
\hline
\end{tabular}

${ }^{1}$ Proequi Melaçada, Guabi ${ }^{\circledR}$ Nutrição Animal; ${ }^{2}$ Valores calculados a partir da seguinte fórmula: $\mathrm{CNE}=[100$ - $(\mathrm{PB}+\mathrm{EE}+\mathrm{MM}+\mathrm{FDN})]$. Fonte: Autores.

Todas as amostras coletadas durante o período experimental foram homogeneizadas e congeladas a $-10^{\circ} \mathrm{C}$ para análises subsequentes. Posteriormente, cada amostra composta foi pré-seca em estufa em ventilação forçada a $55^{\circ} \mathrm{C}$ por $72 \mathrm{~h}$. Após a présecagem as amostras foram moídas em um moinho tipo Willey com peneira de $1 \mathrm{~mm}$ e acondicionada em recipientes plásticos identificados. As análises seguiram a metodologia para matéria seca (MS), proteína bruta (PB), matéria mineral (MM), extrato etéreo (EE), fibra em detergente neutro (FDN) e fibra em detergente ácido (FDA) seguindo a recomendação de AOAC (2012). A fibra em detergente neutro (FDN) e fibra em detergente ácido (FDA) foram determinadas de acordo com da Silva et al. (2020).

A partir disso foram calculados os coeficientes de digestibilidade aparente (CDap) dos nutrientes, de acordo com fórmula descrita abaixo (Garcia et al., 2014):

$$
\operatorname{CDap}(\%)=\frac{(\text { Nutriente consumido }(\mathrm{g})-\text { Nutriente excretado nas fezes }(\mathrm{g}))}{\text { Nutriente consumido }(\mathrm{g})} \times 100
$$

O teor de LIPE ${ }^{\circledR}$ obtido nas fezes, foi estimando para determinação da produção fecal (PF) por meio de espectroscopia de infravermelho de acordo com o descrito por Garcia et al. (2014):

$$
\text { PF na } M S(g)=\frac{\text { LIPE fornecido }(\mathrm{g})}{\frac{A i}{\text { MS total }}} X 100
$$

Onde: $\mathrm{PF}=$ Produção fecal; $A_{i}=$ Relação logarítmica das intensidades de absorção das bandas dos comprimentos de onda a $1050 \mathrm{~cm}^{-1} / 1650 \mathrm{~cm}^{-1}$; MS total = matéria seca fecal total;

O Ai será obtido através da fórmula:

$$
A_{i}=\frac{A 1050}{A 1650}
$$

Sendo que: em que: $\mathrm{A}=\log \frac{I^{\circ}}{I}$, de modo que: $\mathrm{I}^{\mathrm{o}}>$ intensidade; $\mathrm{I}<$ intensidade. 
Ainda foram calculadas as taxas de recuperação fecal (\%) do indicador utilizando-se a fórmula, descrita a seguir (Lanzetta et al., 2009):

$$
\text { Taxa de recuperação fecal }(\%)=\frac{\text { PF estimado pelo indicador }}{\text { PF obtida pela CT }} X 100
$$

Em que :PF estimada pelo indicador = produção fecal estimada utilizando-se o indicador (LIPE $\left.{ }^{\circledR}\right)$.

PF obtida pela CT = produção fecal obtida pelo método da coleta total de fezes.

\section{Análise Estatística}

Os dados utilizados neste estudo foram do tipo quantitativo (Sakomura e Rostagno, 2016; Pereira et al., 2018), e adotouse delineamento experimental foi inteiramente casualizado com três tratamentos e seis repetições, sendo o animal considerado uma unidade experimental. Os coeficientes de digestibilidade foram analisados por ANOVA a 5\% de probabilidade, de acordo com o seguinte modelo estatístico:

$$
\text { Yijk }=\mu+T_{i}+e_{i j k}
$$

Onde: Yijk = respostas variáveis (coeficientes de digestibilidade) $\mu=$ constante geral; $\mathrm{Ti}=$ tipos de tratamento (coleta fecal total; LIPE 3; LIPE 5); eijk = erro aleatório associado a cada observação.

\section{Resultados e Discussão}

Não houve diferença $(\mathrm{P}>0,05)$ entre os métodos de coleta quanto aos coeficientes de digestibilidade da matéria seca, matéria orgânica, extrato etéreo, proteína bruta e hemicelulose (Tabela 2).

\begin{tabular}{|c|c|c|c|c|c|}
\hline \multirow{2}{*}{ Variáveis (\%) } & \multicolumn{3}{|c|}{ Tratamentos } & \multirow{2}{*}{$P$-value } & \multirow{2}{*}{$\mathrm{CV}^{1} \%$} \\
\hline & Coleta total & LIPE 5 & LIPE 3 & & \\
\hline Matéria $\mathrm{Seca}^{2}$ & $60,12 \pm 3,11$ & $60,71 \pm 9,40$ & $59,93 \pm 6,21$ & 0,94 & 10,8 \\
\hline Matéria Orgânica $^{3}$ & $61,62 \pm 4,13$ & $61,95 \pm 8,98$ & $61,58 \pm 5,43$ & 0,98 & 10,3 \\
\hline Extrato Etéreo ${ }^{4}$ & $60,58 \pm 6,32$ & $65,70 \pm 12,52$ & $62,77 \pm 15,55$ & 0,49 & 19,5 \\
\hline Proteína Bruta ${ }^{5}$ & $76,58 \pm 3,36$ & $76,39 \pm 7,42$ & $77,34 \pm 5,80$ & 0,89 & 7,7 \\
\hline $\mathrm{FDN}^{6}$ & $56,62 \pm 3,53 \mathrm{a}$ & $66,80 \pm 2.82 b$ & $68,81 \pm 3,96 b$ & $<0,01$ & 5,41 \\
\hline $\mathrm{FDA}^{7}$ & $35,33 \pm 2,87 \mathrm{a}$ & $49,81 \pm 7,21 b$ & $44,54 \pm 2,48 b$ & $<0,01$ & 10,89 \\
\hline Hemicelulose $^{8}$ & $81,20 \pm 7,47$ & $87,17 \pm 1,08$ & $87,76 \pm 3,27$ & 0,09 & 5,56 \\
\hline $\mathrm{CNE}^{9}$ & $65,75 \pm 1,99 a$ & $73,27 \pm 1,64 b$ & $65,27 \pm 3,74 a$ & $<0,01$ & 3,86 \\
\hline
\end{tabular}

Tabela 2 - Efeito do método de coleta sobre os coeficientes de digestibilidade de dietas para equinos.

${ }^{1} \mathrm{CV}=$ Coeficiente de variação; ${ }^{2}$. Matéria seca; ${ }^{3} \mathrm{MO}=$ Matéria Orgânica; ${ }^{4} \mathrm{EE}=$ Extrato etéreo; ${ }^{5} \mathrm{~PB}=\mathrm{Proteína} \mathrm{Bruta;}{ }^{6} \mathrm{FDN}=$ Fibra em detergente neutro; ${ }^{7} \mathrm{FDA}=$ Fibra em detergente ácido; ${ }^{8} \mathrm{HEM}=$ Hemicelulose $;{ }^{9} \mathrm{CNE}=\mathrm{Carboidratos}$ não estruturais. Fonte: Autores. 
Entretanto o coeficiente de digestibilidade aparente da fibra em detergente neutro e ácido foram maiores $(\mathrm{P}<0,01)$ para os métodos LIPE ${ }^{\circledR}$ três e cinco. O método LIPE 5 estimou maior coeficiente de digestibilidade para o carboidrato não estrutural quando comparado ao método controle (Tabela 2).

A taxa média de recuperação fecal do LIPE 5 dias com 93,75\% e LIPE 3 dias com 94,52\% (Figura 1).

Figura 1 - Recuperação fecal obtida pelos métodos de coleta total de fezes LIPE ${ }^{\circledR}$, com coleta de excretas durante três dias (LIPE 3) ou coleta de excretas durante cinco dias (LIPE 5) na matéria seca.

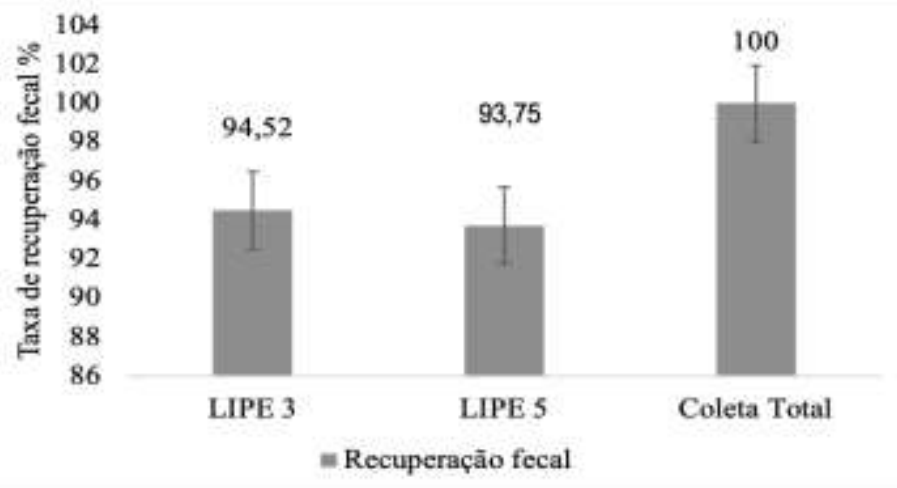

Fonte: Autores.

Observou-se que o método LIPE ${ }^{\circledR}$ (três ou cinco dias de coleta) apresentaram coeficientes de digestibilidade da fibra em detergente neutro e ácido maiores em relação ao método tradicional de coleta de fezes. Ressalta-se que o método LIPE5 demonstrou superestimar a digestibilidade de carboidratos não estruturais. Contudo, estas diferenças observadas na análise das fibras levanta a hipótese de que os métodos LIPE $^{\circledR}$ podem superestimar valores diferentes para estas variáveis quando comparados ao método de coleta total de fezes.

Schaafstra et al. (2018) inferem que o uso de marcadores para estimar a digestibilidade dos nutrientes é menos trabalhoso do que a coleta total de fezes, por isto tem sido indicado. Além disso, no método de coleta total, o controle do quantitativo do fornecimento da dieta, bem como da quantidade de fezes coletadas é essencial. Por este motivo, mais pesquisas para avaliar coeficientes de fibras devem ser realizadas a fim de averiguar esta possível superestimação dos resultados.

Garcia et al. (2014) ao avaliar o método LIPE ${ }^{\circledR}$ com 5 dias de coleta em comparação ao método de coleta total em equinos adultos alimentados com cana de açúcar in natura ou hidrolisada e suplementadas com a levedura Saccharomyces cerevisiae, respectivamente, não encontraram diferenças nas variáveis de digestibilidade analisadas. Em pesquisas realizadas com outras espécies, os resultados obtidos validam o método LIPE ${ }^{\circledR}$, a exemplo da pesquisa realizada por Vasconcellos et al. (2007), utilizando o LIPE ${ }^{\circledR}$ (cinco dias) como indicador externo na determinação da energia metabolizável de alimentos em frangos de corte; e De Moraes et al. (2010), em pesquisa com caprinos alimentados com subprodutos de urucum, avaliando os métodos de estimativa coleta total, LIPE $^{\circledR}$ (cinco dias) e óxido crômico verificaram que não houve diferenças significativas para a produção fecal e para a digestibilidade aparente.

Deste modo, o comportamento da técnica utilizando três ou cinco dias de coleta de fezes para o método da LIPE ${ }^{\circledR}$ demonstraram resultados semelhantes, com exceção ao coeficiente de digestibilidade dos carboidratos não estruturais; permitindo desta forma, o seu uso. Ressalta-se ainda que embora a literatura recomenda o uso de LIPE $^{\circledR}$ durante cinco dias para coletas fecais (Garcia et al., 2014; Moss et al., 2017), foi demonstrado neste trabalho que a utilização deste indicador em coletas por três dias também possibilitou determinar a digestibilidade dos nutrientes da dieta de maneira eficiente, este fato se torna evidente diante dos valores observados na recuperação fecal, que foram sempre próximos de 100\%. Resultados semelhantes aos obtidos neste estudo foram apresentados por Lanzetta et al. (2009), que constataram taxa média de recuperação fecal do LIPE ${ }^{\circledR}$, 
com cinco dias de coleta, de $95,94 \%$ em potras alimentadas com feno de alfafa e concentrado comercial.

Em comparação ao método de coleta total para determinação da digestibilidade de nutrientes em equinos, os métodos que envolvem a utilização de LIPE $^{\circledR}$ (coletas parciais em 3 e 5 dias) podem ser utilizados para a determinação da digestibilidade em eqüinos. Nesta pesquisa, o método LIPE $^{\circledR}$, tanto com 3 quanto com 5 dias de coleta de fezes superestimaram os coeficientes de digestibilidade da fibra em detergente neutro e ácido; enquanto que o método LIPE ${ }^{\circledR}$ com cinco dias de coleta de fezes superestimou o coeficiente de digestibilidade dos carboidratos não estruturais.

\section{Conclusão}

O presente estudo atingiu seus objetivos principais e amplia o entendimento dos nutricionistas e pesquisadores, em especial na área de nutrição de equinos, uma vez que se pode concluir que o método de coleta total de fezes e os métodos com coleta parcial de fezes durante 3 ou 5 dias com utilização de LIPE ${ }^{\circledR}$ pode ser utilizado para se determinar os coeficientes de digestibilidade da matéria seca, matéria orgânica, extrato etéreo, proteína bruta e hemicelulose em equinos de forma confiável.

Por fim, novos estudos devem ser conduzidos a fim de aprofundar questões específicas que, porventura, tenham restado a esclarecer.

\section{Agradecimentos}

Á Universidade Federal de Sergipe pelo apoio financeiro, ao PROMOB, Laboratório de Bacteriologia do Departamento de Ciências Biológicas e da Saúde da Universidade Federal de Sergipe (UFS) e Laboratório de microbiologia do Departamento de Microbiologia (UFMG).

Este estudo foi parcialmente financiado pela Coordenação de Aperfeiçoamento de Pessoal de Nível Superior - Brasil (CAPES) - Código Financeiro 001.

\section{Referências}

AOAC- Association of Official Analytical Chemistry. (2012). Official methods of analysis of AOAC international: current through revision. Gaithersburg: MD.

Da Silva Oliveira, V., da Silva Morais, J. A., Fagundes, J. L., Santana, J. C. S., Muniz, E. N., \& de Lima Valença, R. (2020). Fracionamento de proteína e carboidratos segundo CNCPS de cinco forrageiras irrigadas ou não durante a seca. Research, Society and Development, 9(7), e77973582-e77973582.

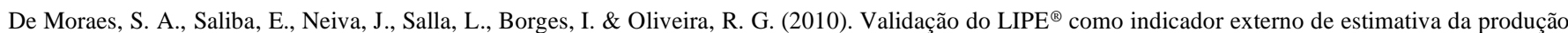
fecal e digestibilidade em caprinos alimentados com subproduto de urucum. In Embrapa Semiárido-Artigo em anais de congresso (ALICE). In: REUNIÃO ANUAL DA SOCIEDADE BRASILEIRA DE ZOOTECNIA, 47, 2010, Salvador: SBZ.

Figueiredo, M. R. P., Saliba, E. O. S., Barbosa, G. S. S. C., Silva, F. A., Nunes, A. N., Silva, C. R. M. \& Moreira, G. R. (2019). Use of indigestible markers to estimate the apparent dry matter digestibility of diets containing a cocoa by-product. Semina: Ciências Agrárias, 40, $2771-2782$.

Garcia, T. R., Rezende, A. S. C., Santiago, J. M., Terra, R. A., Fonseca, M. G., Costa, M. L. L., Lana, A. M. Q. \& Almeida, F. Q. (2014). Digestibility and nutrient intake in Mangalarga Marchador mares supplemented with Saccharomyces cerevisiae during aerobic training. Semina: Ciências Agrárias, 35, 20112018 .

Lanzetta, V. A. S., Rezende, A. S. C., Saliba, E. O. S., Lana, A. M. Q., Rodriguez, N. M. \& Moss, P. C. B. (2009). Validation of Lipe ${ }^{\circledR}$ as method to evaluate the apparent digestibility of nutrients in equines. Revista Brasileira de Zootecnia, 38, 69-74.

Moss, P. C. B., Rezende, A. S. C., Saliba, E. O. S., Lana, A. M. Q., Moura, R. S., Cassou, F., Costa, M. L. L., Freitas, G. P. \& Alves, G. E. S. (2017). Validation of Nanolipe ${ }^{\circledR}$ as method to assess the apparent digestibility of nutrients on horses. Arquivos Brasileiro de Medicina Veterinária e Zootecnia, $69,687-694$.

National Research Council. (2007). Nutrient requirements of beef cattle. Washington DC: National Academy Press.

Pereira, A. S., Shitsuka, D. M., Parreira, F. J. \& Shitsuka, R. (2018). Metodologia da pesquisa científica [recurso eletrônico]. Santa Maria, BR:UFSM, NTE.

Sakomura, N. K. \& Rostagno, H. S. Métodos de pesquisa em nutrição de monogástricos (2016). Jaboticabal, BR: Funep.

Saliba, E. O. S. (2005). Uso de indicadores: Passado, presente e futuro. In: Teleconferência sobre indicadores em nutrição animal. Belo Horizonte: Escola de Veterinária/UFMG. 
Research, Society and Development, v. 10, n. 2, e1310212105, 2021

(CC BY 4.0) | ISSN 2525-3409 | DOI: http://dx.doi.org/10.33448/rsd-v10i2.12105

Silva, A. G. M., Borges, I., Neiva, J. N. M., Rodriguez, N. M., Saliba, E. O. S., Morais, A. S., Silva, J. J., Merlo, F. A., Sousa, T. D. S., Magalhães Junior, L. L., Gonçalves, C. N., Barros V. P. \& Vale R.C.A. (2018). Avaliação do lipe ${ }^{\circledR}$ como indicador externo de digestibilidade em ovinos recebendo dietas com torta de babaçu. Revista Brasileira de Nutrição Animal, 17, 39-44.

Soares, L. F. P., Guim, A., Ferreira, M. A., Modesto, E. C., Batista, A. M. V. \& Monteiro, P. B. S. (2011). Assessment of indicators and collection methodology to estimate nutrient digestibility in buffaloes. Revista Brasileira de Zootecnia, 40, 2005-2010.

Schaafstra, F. J. W. C., Van Doorn, D. A., Schonewille, J. T., Roelfsema, E., Westermann, C. M., Dansen, O., Jacobs, M., Lee, J. Y., Spronck, E. A., Blok, C. \& Hendriks, W. H. (2018). Effect of exercise on apparent total tract digestibility of nutrients and faecal recovery of $\mathrm{ADL}$ and $\mathrm{TiO}_{2}$ in ponies. Animal, 12(12), 2505-2510.

Valentini, P. V., Pancoti, C. G., de Castro Mourão, R., Lima, F. A., Resende, T. Q., Lopes, F. C. F. \& Reis, R. (2016). Utilização do dióxido de titânio (TiO2) como indicador de excreção fecal em estudos de nutrição de ruminantes. PUBVET, 6, 1363.

Vasconcellos, C. H., Veloso, J. A., Saliba, E. O., Baião, N. C. \& Lara, L. J. (2007). Uso da LIPE ${ }^{\circledR}$ como indicador externo na determinação da energia metabolizável de alimentos em frangos de corte. Arquivo Brasileiro de Medicina Veterinária e Zootecnia, 59, 459-465. 